ENCYCLOPÉDIE Encyclopédie berbère

BERBERE

$31 \mid 2010$

31 | Matmora - Meẓrag

\title{
Médications (chez les Touaregs sahéliens)
}

\section{E. Bernus}

\section{OpenEdition}

Journals

Édition électronique

URL : http://journals.openedition.org/encyclopedieberbere/543

DOI : $10.4000 /$ encyclopedieberbere.543

ISSN : 2262-7197

\section{Éditeur}

Peeters Publishers

\section{Édition imprimée}

Date de publication : 30 décembre 2010

Pagination : 4814-4822

ISBN : 978-90-429-2368-3

ISSN : 1015-7344

\section{Référence électronique}

E. Bernus, « Médications (chez les Touaregs sahéliens) », Encyclopédie berbère [En ligne], 31 | 2010, document M80, mis en ligne le 08 octobre 2020, consulté le 12 octobre 2020. URL : http://

journals.openedition.org/encyclopedieberbere/543 ; DOI : https://doi.org/10.4000/ encyclopedieberbere.543

Ce document a été généré automatiquement le 12 octobre 2020

() Tous droits réservés 


\section{Médications (chez les Touaregs sahéliens)}

\section{E. Bernus}

1 La médecine traditionnelle des Touaregs nomades vivant en zone pastorale sahélienne fait appel aussi bien aux végétaux qu'aux minéraux et aux animaux (lait, beurre, graisse, sang, excrétions), sans parler de l'usage du fer rougi au feu pour circonscrire ou inciser une plaie.

\section{Maladies et remèdes}

2 Une distinction connue des Touaregs, mais aussi de bien d'autres populations du monde, oppose le chaud et le froid dans une dichotomie souvent affirmée. Les maladies sont réputées chaudes et froides et, pour les soigner on fait appel à des médicaments ou à des aliments froids pour les premiers, chauds pour les seconds: on soigne une maladie par son contraire. J. Drouin (1993 : 511-534) a tenté d'explorer les champs métaphoriques du «chaud » et du «froid » dans des domaines sémantiques variés, ce qui nous éclaire sur les relations complexes de leur utilisation, au-delà du thème le plus souvent cité concernant les maladies et leurs remèdes.

3 Nos observations ponctuelles chez le Touaregs nigériens ont été confirmées et développées par des études menées chez les Touaregs maliens par des anthropologues et des médecins, par des autochtones et des occidentaux : la synthèse de ces travaux a été publiée par en 1993 (Randall : 4, 127-152). Enfin l'ouvrage récent (2000) de J. Hureiki apporte des éléments nouveaux sur Les médecines traditionnelles touarègues: il illustre une synthèse de ces médecines « qui s'élabore sur la notion d'équilibre considérée ici comme clef de voûte de la structure ; équilibre dans les corps entre le chaud et le froid, et équilibre du corps dans le monde entre Dieu et génies ».

4 Les Touaregs connaissent les maladies contagieuses et savent qu'il faut, autant qu'on le peut, isoler le malade : coryza, rougeole, variole, méningite, coqueluche, etc., sont les principales maladies citées. Éleveurs, les Touaregs ont l'expérience des épizooties qui 
jalonnent leur histoire : peste bovine ou péripneumonie ont périodiquement décimé leurs troupeaux et provoqué ruines, traumatismes et migrations.

La classification des maladies ou des aliments et des médicaments en chauds et froids n'est pas toujours identique d'un informateur à l'autre. Des maladies froides peuvent évoluer vers le chaud, ce qui implique la modification du traitement. Également, l'apparition de maladies chaudes ou froides peut être en rapport avec la saison; certains maux réputés froids tel la méningite est connue sous divers noms, shin akarkor, tenede ta maqqarat, « la grande fièvre » ou ta $n$ iri, « celle du cou », se répandent surtout en saison chaude, alors que le rhume, également froid, est fréquent en saison froide et en saison des pluies. La fièvre, expression d'un mal chaud (tukse), se répand souvent en saison des pluies.

6 La méningite, la variole, la rougeole, la varicelle, maladies contagieuses, exigent qu'on enferme le malade au chaud sous des couvertures, à l'abri de la natte-paravent (asabar), dans un recoin protégé (ifi, efi), le velum de la tente baissé jusqu'au ras du sol. Avant les vaccinations des services de santé, des organismes internationaux ou des ONG, les Touaregs pratiquaient une vaccination contre la variole (erkashik ou bidi), en faisant des scarifications sur le bras, sur lesquelles on introduisait le pus d'un malade (Bernus 1969 : 114).

7 La syphilis (amajer) n'est, en général, pas reconnue comme contagieuse et on ignore comment elle se propage. La blennorragie est appelée tanesmuț, par dérision, puisque ce mot signifie «fraîcheur ». On achète sur les marchés des clous de girofle qu'on pile et donne à boire au malade dans de l'eau.

Les maladies chaudes exigent des aliments froids tels que le lait frais, alors que les maladies froides sont traitées par la viande, les céréales ou le beurre, aliments réputés chauds. Pour les maladies chaudes, on lave le corps avec de l'eau qui est un élément froid. "Le sang et l'eau sont les éléments essentiels de la classification en chaud et froid, et c'est sans doute par leur effet sur ces fluides hors norme que les remèdes sont classés ». La présence du sang là où il ne devrait pas se trouver (saignement de nez par exemple) est signe de chaleur. "S'il y a manque de sang là où il devrait en avoir, c'est (signe de) froid. Qu'il y ait de l'eau (ou des substances aqueuses) là où il ne devrait pas y en avoir est un signe de froid. » (Randall 1993 : 140-141).

D'après Ag Hamahady, cité par Hureiki (2000: 74), les maladies chaudes peuvent être conçues comme aigues et bénignes, alors que les maladies froides sont graves et chroniques.

\section{Soins externes}

Plaies : C'est, bien entendu, sur les plaies, sur les brûlures, sur les infections cutanées de tout ordre - furoncles, panaris, ulcères - voire sur les morsures de serpent ou de scorpion, que des soins utilisent largement la pharmacopée.

11 Pour une plaie (abûs, abuyes) ouverte ou un abcès ouvert, on cherche des feuilles d' aboragh (Balanites aegyptiaca), arbre très commun au sud du Sahara et des noyaux de dattes : on brûle ces dernières dans le feu et on grille les feuilles sèches du Balanites sur un morceau de poterie posé sur des braises. On réduit en poudre le tout dans un petit mortier et on recueille la poudre par vannage. Sur de la graisse de dromadaire ou de 
mouton contenue dans un chiffon pressé pour en exprimer le corps gras comme une pommade, on répand cette poudre ${ }^{1}$.

Pour un furoncle ou un petit abcès que l'on veut faire crever, on cherche du tabac (que tout le monde possède en raison de l'usage général de la chique), de l'écorce de l'agar (Maerua crassifolia), et du blé si on en a, car chez les Touaregs méridionaux, le blé est une céréale beaucoup moins consommée que le mil*. On pile ces deux ou trois produits dans un petit mortier, on verse dessus du lait de brebis et on étale le produit sur la partie douloureuse. Si le lait manque, on insalive la mixture et on en couvre le furoncle.

Les plaies produites par le fer d'une lame, d'un couteau ou d'une épée sont soignées avec du lait de chèvre chauffé que l'on verse sur la plaie. Ensuite, des feuilles de Balanites aegyptiaca carbonisées sont mêlées à du tabac et au fruit d'un arbre du sud acheté sur les marchés et non identifiés ; la mixture est réduite en poudre avant d'être versée sur la plaie qui a reçu du lait.

Le plus souvent les feuilles du Balanites aegyptiaca et du tabakat (Ziziphus lotus), servent de cataplasme sur les plaies et les furoncles. On utilise aussi le «latex" du tirza ou torsha (Calotropis procera) pour désinfecter les plaies (Ag Sidiyene $1996: 50$ ).

Plusieurs types d'onguents nous ont été indiqués pour les plaies purulentes:

- cendre de l'herbe teberemt (Cymbopogon schoenanthus) mêlée à de la graisse de chèvre fondue ;

- sucre et charbon de bois pilés ;

- tabac et natron* mélangés ;

- écorce souple de l'afagag (Acacia tortilis subsp. raddiana), séchée et réduite en poudre ;

- gomme de l'orof (Acacaia seyal) écrasée ;

- gomme-résine de l'adaras (Commiphora africana) liquide, sur les plaies profondes, particulièrement purulentes.

$\mathrm{Au} \mathrm{XIX}^{\mathrm{e}}$ siècle et encore au début du XXe , les blessures par le fer des lances et des épées étaient fréquentes; si elles existent toujours, les plaies les plus nombreuses sont dues aujourd'hui aux branches épineuses qui traînent sur le sol ou aux souches des arbres heurtées en marche ou encore au frottement des cordes trop vite dévidées au puits.

\section{Maux divers}

Le rhume (amaezla) est fréquent en saison froide où le thermomètre descend au-dessous de zero la nuit. On égorge un cabri ou un agneau bien gras que l'on fait cuire avec du piment et du sel. Le patient mange cette viande et met la tête sous une couverture jusqu'à ce que la sueur sorte de tout son corps.

18 L'irritation des yeux, l'ophtalmie, est très fréquente, particulièrement en saison sèche, lorsque le vent du nord, l'harmattan, souffle, chargé d'une fine poussière. Les yeux sont irrités, enflés, rougis ; les paupières sont collées au réveil. Les feuilles fraîches du tamat (Acacia ehrenbergiana) sont mâchées et posées autour de l'œil. Les feuilles du tiggart (Acacia nilotica) bouillies, mises dans un sachet sont posées sur l'œil souffrant de conjonctivite. On peut aussi mettre des grains de mil*, enele (Pennisetum), dans de l'eau portée à ébullition et on lave les yeux avec cette décoction.

19 Les maux de tête suscitent de nombreux traitements. On introduit dans les narines du tabac en poudre venu du nord de l'Ahaggar. D'autres y introduisent le «latex » du 
Calotropis procera, arbre très commun qui a tendance à se multiplier lors des déficits pluviométriques prolongés, aux dépens des acacias et d'autres arbres plus utiles.

Les amandes légèrement torréfiées du fruit du Balanites aegyptiaca servent à se protéger les lèvres et à soulager les gerçures des pieds Enfin les graines de ricin (Ricinus communis), également torréfiées servent à soigner la galle.

La maladie de l'oreille, l'otite, fait suppurer. Si c'est un enfant, on perce le lobe de l'oreille avec une épine que l'on laisse en place. Si s'agit un adulte, on salive des feuilles de tiggart (Acacia nilotica) que l'on crache dans l'oreille du malade ou, à défaut, on utilise du tabac.

La carie dentaire est calmée en mettant de la graisse de margouillat dans la cavité : une aiguille servant à coudre le velum des tentes (tistant) est chauffée au rouge pour faire fondre la graisse qui s'introduit dans la carie et soulage le patient.

Le gonflemant du cou (tabayoq) rend le malade fiévreux. On lui fait mâcher des feuilles de tamat (Acacia ehrenbergiana) et de tabakat (Ziziphus lotus) que l'on prend soin de cueillir du côté ombragé de l'arbre (à l'Ouest le matin, à l'Est le soir). Le malade mâche les feuilles et avale sa salive.

La congestion, le coup de sang (azeni), rougit les yeux. On pratique trois scarifications (shigiyaz, sing. tigiyest) sur la tempe gauche et trois sur la tempe droite. On met de l'antimoine (tazolt) dans les yeux que le malade a tendance à frotter.

\section{Accidents, chutes, fractures, douleurs}

La chute, en particulier la chute de dromadaire, provoque des douleurs généralisées s'il n'y a ni fracture, ni entorse. On met au chaud le blessé, sous des couvertures, et on lui donne de la nourriture chaude, viande avec sel et piment, et bouillon dans lequel la viande a cuit. Sous les couvertures, le sang circule, et on pense éviter des « poches de sang » (hématomes) dans une partie quelconque du corps.

La foulure, l'entorse, sont soignées par application sur le membre atteint de la terre provenant d'une termitière (erraman), mise à chauffer sur le feu dans un morceau de vieille poterie. Un autre traitement consiste à faire des scarifications (shigiyaz) sur le membre atteint, à l'aide de la lame d'un couteau tranchant (elkindarni) dont on se sert pour raser la tête.

La fracture est réduite par un massage destiné à remettre en place l'os brisé. On fixe des attelles au membre avec des cordes, et on laisse immobile le maladeque l'on nourrit avec de la pâte de mil (eshink) et de la viande.

Pour les douleurs de poitrine, des difficultés à respirer, ressenties souvent après un effort violent, pour soulever un objet très lourd par exemple, on creuse un trou dans lequel on allume un feu : puis on retire les braises, on balaie l'excavation et on la tapisse de l'herbe teberemt (Cymbopogon schœnanthus). On dépose ensuite du sable imprégné d'urine de chèvre sur lequel on porte des branches de l'arbuste tadant (Boscia senegalensis). Le malade est allongé dans le trou après avoir absorbé une nourriture très chaude : on le couvre de sable et seule sa tête émerge. Une tente est dressée au-dessus du malade qui transpire et guérit : on enduit son corps de beurre cuit et le lendemain on le lave à l'eau chaude. 
les maux de reins, et sans doute pour les lumbagos et les zonas, on a recours à des massages. Pour les rhumatismes (iselimen) qui rendent douloureux les articulations genoux, poignets, coudes -, on peut faire des massages pour faire disparaitre les grosseurs que l'on tient pour cause du mal.. On pose souvent également un anneau en cuivre rouge aux chevilles ou aux poignets. Enfin, on peut appliquer de la graisse d'autruche ou frotter le corps, tôt le matin, avec du lait de vache ou de brebis.

\section{Médications internes}

Parmi les maux qui font appel aux végétaux par usage interne, la fièvre, de quelque origine qu'elle soit, peut être citée. Elle attaque souvent les nomades en saison des pluies ou en début de saison sèche (octobre), lorsque les mares sont encore pleines : il s'agit en général de fièvres paludéennes liées à la présence des moustiques.

31 Parmi les traitements, on donne au malade le lait de l'aghajera, plat composé de lait, de mil pilé cru et parfois de dattes, de sucre et de fromage, additionné de piment. On essaye de faire vomir le malade et de faire cracher la bile (tazefnent) qui donne son nom à l'affection. On fait alors une infusion d'agerger (Cassia acutifolia), plante buissonnante, fréquente dans le lit des oueds. On peut aussi piler les épis de l'afazu, (Panicum turgidum), herbe vivace, en touffes, qui colonise les dunes sud-sahariennes, enfermer le résidu dans un chiffon et verser dessus de l'eau chaude; l'infusion est donnée au patient. Enfin, des feuilles du Balanites aegyptiaca sont infusées dans un chiffon de la même manière.

Lorsque les voies respiratoires ou la gorge sont chargées de secrétions qui gênent la respiration, des feuilles de tezaq (Salvadora persica) pilées et additionnées d'eau chauffée par des cailloux mis au feu, facilitent l'expectoration (Ag Sidiyene 1996 : 84).

Les maux de ventre font aussi appel aux végétaux par voie interne. Le ventre gonflé, douloureux est attribué à un excès de nourriture ou à l'absorption d'aliments incompatibles : mil et riz, thé et lait par exemple ou encore « de la prise successive de lait de chamelle et de lait de brebis, d'un œuf dur et de lait aigre (lait mélangé...)» (Drouin 1990: 70). On donne alors au malade un premier verre de thé, amer et peu sucré, qui soulage la douleur. Parfois on cherche à purger le malade en lui donnant une préparation composée de terre salée et d'une émulsion de beurre dans de l'eau. On purge aussi avec une infusion d'agerger (Cassia acutifolia) enfermée dans un chiffon, dans une eau légèrement salée : le patient boit cette infusion en ne s'alimentant qu'avec du lait pendant trois jours ; le quatrième, il mange de la viande pilée avec du beurre.. On utilise aussi une poire à lavement faite d'une petite outre à l'orifice de laquelle on place une canule en os: on prépare alors un liquide dans lequel on met à tremper la partie souple de l'écorce du Balanites aegyptiaca. On filtre on chauffe une partie du liquide que l'on ajoute à la partie non chauffée avec du beurre cuit, du sel ; ce liquide est utilisé pour le lavement.

34 Les coliques sont traitées par absorption de diverses mixtures: savon noir de Nigeria dissous dans de l'eau ; mélange de mil pilé avec du sel dans de petites boules que l'on avale avec de l'eau ; enfin les feuille du basilic (Ocimum basilicum), buisson odoriférant, pilées et bues avec du lait caillé.

35 La constipation, le ventre gonflé, sont traités par des lavements à base d'urine de dromadaire, de graines tagellet, (Citrullus colocynthis), coloquinte amère, et de l'écorce

Encyclopédie berbère, 31 | 2010 
souple du tamat (Acacia ehrenbergiana) et de l'aboragh (Balanites aegyptiaca): on enferme le tout dans un tissu au travers duquel on fait passer l'eau qui sert au lavement.

\section{Choix des intervenants}

Dans certains cas, on a recours aux soins d'une personne choisie pour des caractéristiques particulières. Pour les massages destinés à soulager les maux de reins, lumbagos ou zonas, on cherche une femme ayant donné naissance à des jumeaux (ikinewen). Il en va de même pour les rhumatismes: la femme demande au patient depuis quand il est victime de la crise et donne la date de naissance de ses jumeaux : il y a comme une connivence, un jeu, entre les deux parties qui doit être bénéfique au malade.

Pour tafuré, affection qui provoque la décoloration de la peau et donne à celui qui en est atteint un aspect tacheté que l'on appelle azeghraf, comme les chameaux pies des Kel Geres du sud du Niger, qui participent aux caravanes du sel de Bilma. Bien que cette maladie ne provoque aucune ulcération de la peau, on entreprend un traitement. On fait appel à une personne qui possède un lien de parenté spécial avec le patient, un cousin croisé, homme ou femme, abobaz ou tubobaz, c'est-à-dire enfants d'un frère ou d'une sœur, qui est réputé avoir la meilleure chance d'apporter la guérison : celui-ci prend un morceau de poterie noirci au feu, frotte la peau décolorés jusqu'à la faire saigner et saupoudre la plaie avec la cendre de la paille d'herbe (Panicum turgidum) ou de mil. Le cousin croisé peut aussi lécher la partie dépigmentée de la peau.

\section{Conclusion}

Toutes les utilisations de cette riche pharmacopée s'appuient sur une grande expérience et sur une connaissance qui est souvent celle des femmes âgées. Ce sont des traditions orales transmises de générations en générations qui ont établi ces usages thérapeutiques.

La magie et la religion jouent un grand rôle dans cette médecine et de nombreux marabouts sont consultés pour leur savoir : ils sont recherchés pour soigner tous ceux qui sont aux prises avec les kel esuf toujours redoutés dans des zones précises: emplacements de campements abandonnés, terriers, cimetières ou à proximité d'arbres tel le Maerua crassifolia. Pour le traitement de ces maladies mentales, attribuées aux kel esuf, on fait appel aux marabouts et aussi à des traitements par le son du tambour (tende) et de la vièle ( $a m z a d$ ), recours à des pratiques païennes issues du fond des temps.

«Les Touaregs comme les Maures adoptèrent les génies en tant que facteur causal responsable de leurs malheurs et de leurs maux [...]. Tandis qu'ils ne retiennent de la causalité naturelle que deux principes (chaud, froid) » (Hureiki, $2000: 17$ ). 


\section{BIBLIOGRAPHIE}

AG SIDIYENE E., 1996 - Des arbres et des arbustes spontanés de l'Adrar des Iforas (Mali). Étude ethnolinguistique et ethnobotanique, avec la collaboration d'Edouard Le Floc'h et d'Edmond Bernus, Paris, ORSTOM / CIRAD : $137 \mathrm{p}$.

BERNUS, Ed. 1969 - « Maladies humaines et animales chez les Touaregs sahéliens », Paris, Journal de la Société des Africanistes, XXXIX, 1 : 11-137.

BERNUS, Ed., 1993 - Touaregs nigériens. unité culturelle et diversité régionale d'un peuple pasteur. Paris, L'Harmattan. $1^{\text {ère }}$ édition 1971, Mémoire ORSTOM nº $94: 509$ p.

DROUIN, J., 1990 - « Perception sectorielle et synesthésie. Essai sur les données touarègues ». Paris, Littérature Orale Arabo-Berbère 21 (1990) : 61-92.

DROUIN J., 1993 - « Esthétique et axiologie. Les concepts » chaud « et » froid « en touareg ». Paris, À la croisée des études libyco-berbères. Mélanges offerts à Paulette Galand et Lionel Galand, édité par J. Drouin et A. Roth, Librairie orientaliste Paul Geuthner : 511-534.

GAST M., 2000 - Moissons du désert. Utilisation des ressources naturelles au Sahara central, Paris, Ibis Press : $160 \mathrm{p}$.

HUREIKI J., 2000 - Les médecines touarègues traditionnelles. Approche ethnologique, préface A. Bourgeot, Karthala : $188 \mathrm{p}$.

NICOLAISEN J., 1961 - « Essai sur la religion et la magie touarègue », Copenhague, Folk, 1961 (3) :

113-162.

NICOLAISEN J., 1963 - Ecology and culture of the Pastoral Tuareg. With particular reference to the Tuareg of Ahaggar et Ayr. Copenhagen, National museets skrifter : 548 p.

NICOLAS, F., 1950 - Tamesna. les Ioullemmeden de l'Est ou Touareg Kel Dinnik. Paris, Imprimerie Nationale : $279 \mathrm{p}$.

PRASSE K. G., 1964 - Manuel de grammaire touarègue, IV-V, Nom, Copenhague, Akademisk Forlag : $440 \mathrm{p}$.

RANDALL S. C., 1993 - « Le sang est plus chaud que l'eau (médecine tamacheq) », p. 127-152, in Se soigner au Mali, ss. la dir. de J. Brunet-Jailly, Karthala-ORSTOM : 342 p.

RANDALL S. C., 1993 - « Blood is hotter than water : popular use of hot and cold in Kel Tamasheq illness management ", in Social Science and Medecine, Oxford, New York, Seoul, Tokyo, Pergamon Press : vol. $36, \mathrm{n}^{\circ} 5$ : 673-681.

SECCI Dr J., 2000 - Guide médical Français-Tamasheq, Traduction en tamasheq d'Alhassane ag Solimane, avec la participation d'Aminane Abdoussamed. Préface du Professeur Marc Gentilini, Paris, L'Harmattan : 398 p.

WALETT FAQQI F. - 1993, Isefran. Maladies et soins en milieu touareg. Éditions CRMT / PSMTM, B.P. 23

Bandiagara (Mali), C.P. 1435 Perugia (Italie) : 62 p. 


\section{NOTES}

1. Il existe un terme, tebet, signifiant "appliquer un médicament en poudre sur une plaie » (Foucauld, 1951-52 : IV, 1879) et un terme pour désigner un médicament en poudre, asättäbu plur. isetteba (Prasse, 1964 : IV-V, 165).

INDEX

Mots-clés : Ethnologie, Ethnographie, Maladie, Mali, Sahel, Santé, Touareg 